\title{
Comparative study of four reactions at onset of pre-equilibrium emission
}

\author{
Magda Cicerchia $^{1,2, *}$, Fabiana Gramegna $^{2}$, Daniela Fabris ${ }^{3}$, Tommaso Marchi ${ }^{2}$, Marco Cinausero $^{2}$, Giorgia Mantovani ${ }^{1,2}$, \\ Meltem Degerlier ${ }^{4}$, Sandro Barlini ${ }^{5}$, Maurizio Bini ${ }^{5}$, Mauro Bruno ${ }^{6}$, Antonio Caciolli ${ }^{1,3}$, Alberto Camaiani ${ }^{5}$, Giovanni \\ Casini $^{5}$, Natalia Cieplicka-Orynczak ${ }^{7}$, Gianmaria Collazuol ${ }^{1,3}$, Michela D'Agostino ${ }^{6}$, Daniele Dell'Aquila ${ }^{8}$, Bogdan $^{2}$ \\ Fornal $^{7}$, Catalin Frosin ${ }^{5}$, Nicla Gelli ${ }^{5}$, Diego Gruyer ${ }^{9}$, Vladimir Kravchuk ${ }^{10}$, Silvia Leoni ${ }^{11}$, Ivano Lombardo ${ }^{12}$, Daniele \\ Mengoni ${ }^{1,3}$, Luca Morelli $^{6}$, AlessandroOlmi ${ }^{5}$, Pietro Ottanelli ${ }^{5}$, Gabriele Pasquali ${ }^{5}$, Silvia Piantelli ${ }^{5}$, Giacomo Poggi ${ }^{5}$, \\ Simone Valdré
}

${ }^{1}$ Dipartimento di Fisica e Astronomia dell'Università di Padova, Padova, Italy.

${ }^{2}$ INFN Laboratori Nazionali di Legnaro, Legnaro (PD), Italy.

${ }^{3}$ INFN Sezione di Padova, Padova, Italy.

${ }^{4}$ Science and Art Faculty, Physics Department, Nevsehir Haci Bektas Veli Univ., Nevsehir, Turkey.

${ }^{5}$ INFN, Florence Department and University, Physics and Astronomy Department - Florence, Italy.

${ }^{6}$ INFN, Bologna Department and University, Physics and Astronomy Department - Bologna, Italy.

${ }^{7}$ Institute of Nuclear Physics, Polish Academy of Sciences Krakow, Poland.

${ }^{8}$ MSU Department of Physics and Astonomy - Michigan USA.

${ }^{9}$ Grand Accélérateur National d'Ions Lourds, 14076 Caen, France.

${ }^{10}$ National Research Center "Kurchatov Institute" Moscow, Russia.

${ }^{11}$ INFN, Milan Department and University, Physics and Astronomy Department - Milan, Italy.

${ }^{12}$ INFN, Catania Department - Catania, Italy.

\begin{abstract}
The study of the emitted particles, comparing pre-equilibrium and thermal components, is a useful tool to examine the nuclear structure of emitters. Possible clustering effects, which may change the expected decay chain probability, could be highlighted on the competition between different reaction mechanisms. The NUCL-EX collaboration (INFN, Italy) has carried out an extensive research campaign on pre-equilibrium emission of light charged particles from hot nuclei. In this framework, the reactions ${ }^{16} \mathrm{O}+{ }^{30} \mathrm{Si},{ }^{18} \mathrm{O}+{ }^{28} \mathrm{Si},{ }^{19} \mathrm{~F}+{ }^{27} \mathrm{Al}$ at $7 \mathrm{AMeV}$ and ${ }^{16} \mathrm{O}+{ }^{30} \mathrm{Si}$ at $8 \mathrm{AMeV}$ have been carried out using the GARFIELD+RCo array at Legnaro National Laboratories. Some anomalies in the $\alpha$-particle emission channels have been evidenced in the measurement reported above, showing in an exclusive way the observed effects related to the entrance channels. The experimental results are compared to model prediction, for which the same filtering and complete event selection have been applied.
\end{abstract}

\section{Introduction}

In the past decades, improvements in the knowledge of the incomplete fusion reactions have been achieved [1-3]; nevertheless, a complete understanding of reaction mechanisms associated with the emission of the particles prior the thermalization, like break-up and preequilibrium emissions, is still missing. Frequently, the term incomplete fusion has been employed to indicate that, somehow, particles are lost from the projectile and/or target before complete thermalization, which occurs up to the fusion of the remnants.

At bombarding energy above $10 \mathrm{AMeV}$, even-though usually a complete thermalization occurs, the pre- equilibrium particle emission becomes an increasingly important process as a function of the bombarding energy; such particles are forward focused and emitted in the very early stages of the collision before the attainment of full statistical equilibrium of the compound system $[4,5]$. Even in the energy region 5-10 AMeV, fast emission processes have been observed when the structure of the projectile plays an important role in the interaction with the target. This projectile break-up mechanism [6-9], as well as the pre-equilibrium, influences the following formation and decay of the hot source.

\footnotetext{
* Corresponding author: cicerchia@lnl.infn.it
} 
Since several years, the NUCL-EX collaboration (INFN, Italy) has carried out an extensive research campaign on pre-equilibrium emission of light charged particles from hot nuclei [10-14].

\section{The Experiment}

In this framework, four reactions ${ }^{16} \mathrm{O}+{ }^{30} \mathrm{Si}$ at 7 and 8 $\mathrm{AMeV},{ }^{18} \mathrm{O}+{ }^{28} \mathrm{Si}$ at $7 \mathrm{AMeV}$ and ${ }^{19} \mathrm{~F}^{27} \mathrm{Al}$ at $7 \mathrm{AMeV}$, forming the same compound nucleus $\left({ }^{46} \mathrm{Ti}^{*}\right)$ in case of complete fusion, were investigated [15,16]. The experiment was carried out at Legnaro National Laboratories (INFN-LNL, Italy) using the GARFIELD+RCo $4 \pi$ array for charged particles, fully equipped with digital electronics [17]. For sake of comparison, the beam velocity was kept constant (7 $\mathrm{AMeV}$ ) for the three reactions, since the abundance of pre-equilibrium particles is demonstrated to be dependent on it [18]: in such a way, the non-equilibrium processes are expected to be almost the same. The reaction ${ }^{16} \mathrm{O}+{ }^{30} \mathrm{Si}$ has been also measured at a beam energy of $8 \mathrm{AMeV}$ to populate the ${ }^{46} \mathrm{Ti}^{*}$ at the same excitation energy of the ${ }^{18} \mathrm{O}+{ }^{28} \mathrm{Si}$ at $7 \mathrm{AMeV}$ to obtain a similar statistical component. The main characteristics of studied reactions, in case of complete fusion, are reported in Table I.

Table 1. Summary of the main characteristics of the studied reactions.

\begin{tabular}{|c|c|c|c|c|}
\hline $\begin{array}{c}\text { Entrance } \\
\text { Channel }\end{array}$ & $\begin{array}{c}\text { Mass } \\
\text { asymmetry }\end{array}$ & $\begin{array}{c}\mathrm{E}_{\text {lab }} \\
(\mathrm{AMeV})\end{array}$ & $\mathrm{CN}$ & $\begin{array}{c}\mathrm{E}_{\mathrm{CN}}^{*} \\
(\mathrm{MeV})\end{array}$ \\
\hline${ }^{16} \mathrm{O}+{ }^{30} \mathrm{Si}$ & 0.30 & 7 & ${ }^{46} \mathrm{Ti}$ & 88.0 \\
\hline${ }^{16} \mathrm{O}+{ }^{30} \mathrm{Si}$ & 0.30 & 8 & ${ }^{46} \mathrm{Ti}$ & 98.4 \\
\hline${ }^{18} \mathrm{O}+{ }^{28} \mathrm{Si}$ & 0.22 & 7 & ${ }^{46} \mathrm{Ti}$ & 98.5 \\
\hline${ }^{19} \mathrm{~F}+{ }^{27} \mathrm{Al}$ & 0.17 & 7 & ${ }^{46} \mathrm{Ti}$ & 103.5 \\
\hline
\end{tabular}

\section{The Data Analysis}

The complete analysis has been performed on an eventby-event basis; a detail description of this analysis is given in Ref. [15]. In the present work, we focus the attention on the complete events $\left(Z_{\text {tot,detect }}=Z_{\text {projectile }}+\right.$

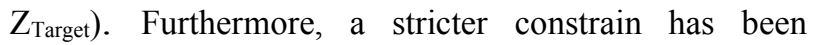
imposed on data: we select the emission of light charged particles (LCP) in coincidence with one and only one fragment with $Z_{\text {frag }}>5$ (ER); such events correspond to almost central collisions.

When we compare the experimental global observables (e.g. charge distribution and multiplicities of the emitted light charged particles) of the 4 reactions, a clear dependence of the yields on the excitation energy of the compound nucleus $\left(\mathrm{E}_{\mathrm{CN}}\right)$ and on the center of mass velocity $\left(\mathrm{v}_{\mathrm{cm}}\right)$ is observed.
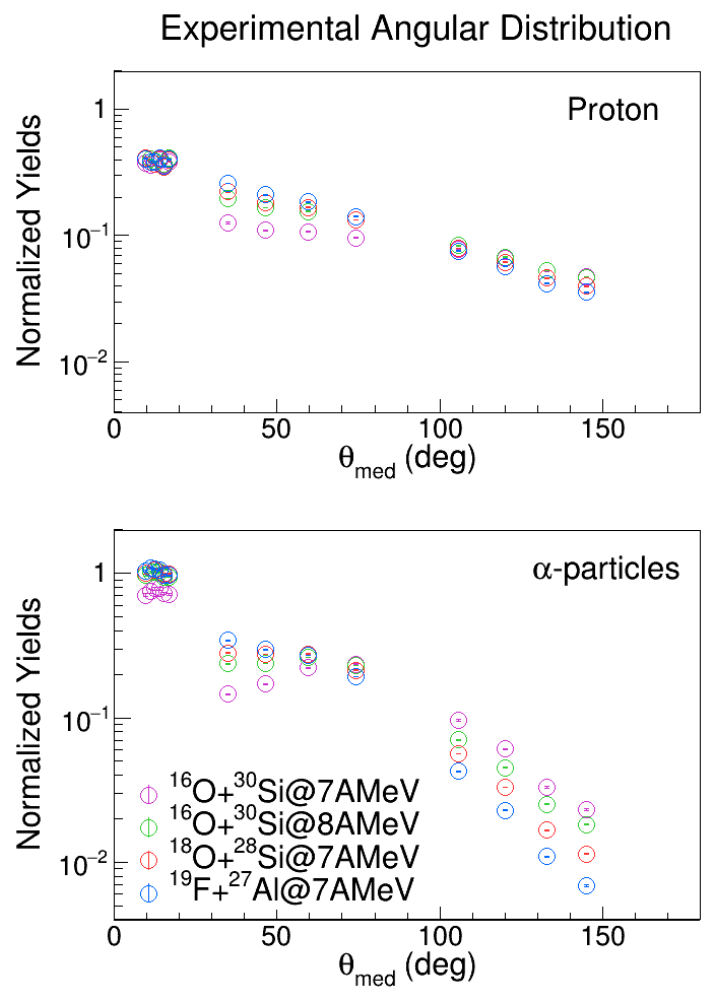

Fig. 1. Experimental angular distribution of protons (upper panel) and $\alpha$-particles (lower panel) for the four studied reactions. The distributions are normalized to the number of complete events. The error bars are inside the experimental points.

Similarly, the experimental angular distribution depends on $v_{\mathrm{cm}}$ (Fig. 1), even-though a strong over-production of LCP (more important in the case of the $\alpha$-particles) appears at very forward angles $\left(8.8^{\circ}-17.4^{\circ}\right)$.

\subsection{The Statistical Code}

In order to have a theoretical feedback, the experimental data have been compared with simulations performed with the statistical code GEMINI ${ }^{++}$[19], which describes the decay of the excited compound nucleus. The simulated events were filtered through a software replica of the experimental setup and, then, selected in the same way of the experimental events [15].

In Fig. 2, the simulated angular distributions of protons and $\alpha$-particles are shown. The experimental trend and dependence on $\mathrm{v}_{\mathrm{cm}}$ are reproduced in the angular region from $29.5^{\circ}$ to $150.5^{\circ}$, while at very forward angles $\left(8.8^{\circ}\right.$ $17.4^{\circ}$ ) significant deviations from the experimental 
$\mathrm{GEMINI}^{++}$simulated Angular Distribution
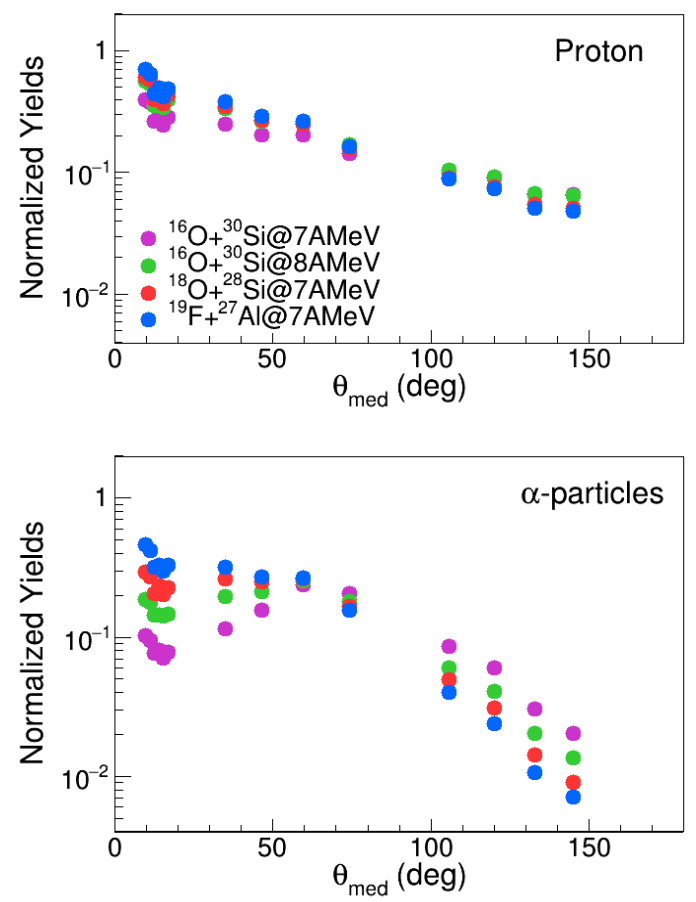

Fig. 2. GEMINI ${ }^{++}$simulated angular distribution of protons (upper panel) and $\alpha$-particles (lower panel) for the four studied reactions. The distributions are normalized to the number of complete events.

angular distribution are observed. Quantitatively, the divergences between the simulated and experimental angular distributions are shown in Fig. 3, where the ratios between the experimental and simulated $\alpha$-particles yields are shown as a function of the detection angle of the particles. As it can be observed, in the angular region from $29.5^{\circ}$ to $150.5^{\circ}$, the experimental yields of $\alpha$ particles are compatible with a statistical emission from the compound nucleus.

Otherwise, the observed overproduction of experimental $\alpha$-particles is present at very forward angles $\left(8.8^{\circ}-17.4^{\circ}\right)$. According to the literature [18], such over-production of forward focused $\alpha$-particles should be related to fast emissions from non-equilibrium processes, characterizing the early stage of the reactions; it depends on both the entrance channel mass asymmetry $(\eta)$ and the beam velocity $\left(\mathrm{v}_{\text {beam }}\right)$. However, in our case we observe some peculiar behaviour: the $\alpha$-energy spectra are reproduced
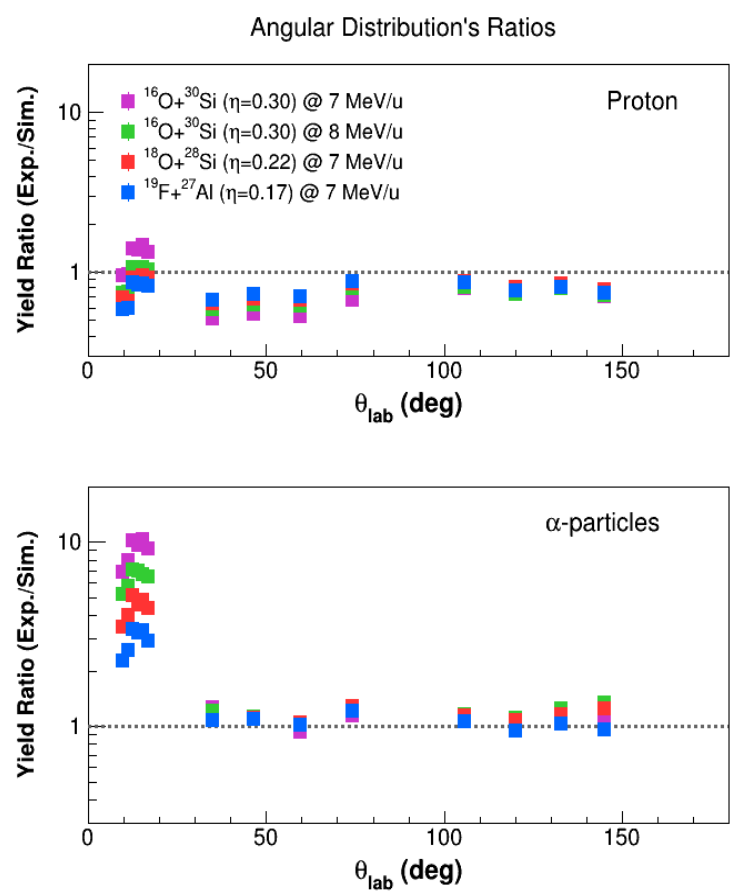

Fig. 3. Ratio of experimental and simulated proton (upper panel) and $\alpha$-particles (lower panel) yields as a function of the detection angle of the particles for the four studied reactions.

in shape and the forward missing $\alpha$-yields are distributed over all the possible energies [15].

Moreover, at variance with what expected, when we compare the results of the two reactions with the same $\eta$ (same entrance channel: ${ }^{16} \mathrm{O}+{ }^{30} \mathrm{Si}$ ), we observe a larger ratio (Exp./GEMINI ${ }^{++}$) of forward emitted $\alpha$-particles yields (Fig. 2) in the case of the reaction at the lower $\mathrm{V}_{\text {beam }}$ $(7 \mathrm{AMeV})$. When comparing the three reactions with the same $\mathrm{v}_{\text {beam }}(7 \mathrm{AMeV})$, an increase of the ratio at forward angles is seen as the $\eta$ increases. Despite the small difference in $\eta$, this effect seems to be larger than expected, suggesting that the internal structure of the interacting nuclei may also play an important role. For the studied systems, the major part of the forward peaked $\alpha$ particles is correlated to the exclusive channel with larger $\mathrm{Z}$ of residues ; in particular, in the Ca-residue exit channels, a strong inversion of population of $1 \alpha+\mathrm{xn}$ and $2 p+x n$ channels is observed with respect to GEMINI ${ }^{++}$. 


\section{Conclusion}

We analysed complete events of four reactions having different entrance channels $(\eta)$ and/or different beam velocity and then different $\mathrm{v}_{\mathrm{cm}}$. The observed differences among the four reactions can be ascribed to either entrance channels or structure properties of the reacting partners. Strong dissimilarities between experimental data and statistical model simulation are highlighted especially related to cluster-emission probability. In particular, enhanced pure $\alpha$-emission has been observed, which, in some cases, become the dominant emission channel at variance with statistical model predictions.

\section{References}

1. K. A. Griffioen et al., Phys. Rev. C 372502 (1988).

2. J. Gomez del Campo et al., Phys. Rev. C 60021601 (1999); 53222 (1996).

3. M. Blann, Phys. Rev. C 311245 (1985).

4. S. J. Luke, Phys. Rev. C 48857 (1993).

5. J. Cabrera, Phys. Rev. C 68 034613-1 (2003).

6. X. Campi et al., Phys. Lett. B 1428 (1984).

7. J. Pouliot et al., Phys. Lett. B 299210 (1993).

8. D. Shapira et al., Phys. Rev. C 552448 (1997).

9. W. D. M. Rae et al., Phys. Rev. C 30158 (1984).

10. T. Marchi et al. - Nuclear Particle Correlations And Cluster Physics - Ch. 20507 (2017);

11. L. Morelli et al., Journ. Of Phys. G 41075107 (2014); 075108 (2014).

12. D. Fabris et al., PoS X LASNPA 061 (2013).

13. V.L. Kravchuk, et al. EPJ WoCs 210006 (2010).

14. O. V. Fotina et al., Int. Journ. Mod. Phys. E 191134 (2010).

15. M. Cicerchia, PhD thesis (2018), University of Padova (Italy).

16. M. Cicerchia, IL NUOVO CIMENTO 41 C (2018) 98.

17. M. Bruno et al., EPJ A 49128 (2013).

18. P. E. Hodgson, Phys. Rep. 3741 (2003).

19. R. J. Charity, Phys. Rev. C 82014610 (2010); D. Mancusi et al., Phys. Rev. C 82044610 (2010). 